\title{
Ebselen abolishes vascular dysfunction in influenza A virus-induced exacerbations of cigarette smoke-induced lung inflammation in mice
}

\author{
Kurt Brassington ${ }^{1}$, Stanley Chan ${ }^{1}$, Simone De Luca ${ }^{1}$, Aleksandar Dobric ${ }^{1}$, Kevin Mou ${ }^{1}$, \\ Huei Seow ${ }^{1}$, Osezua Oseghale ${ }^{1}$, Steven Bozinovski ${ }^{1}$, Stavros Selemidis ${ }^{1}$, and Ross Vlahos ${ }^{1}$ \\ ${ }^{1}$ RMIT University
}

October 26, 2021

\begin{abstract}
Background and Purpose: People with COPD are susceptible to respiratory infections which exacerbate pulmonary and/or cardiovascular complications, increasing their likelihood of death. The mechanisms driving these complications remain unknown but increased oxidative stress has been implicated. Here we investigated whether influenza A virus (IAV) infection, following chronic cigarette smoke (CS) exposure, worsens vascular function and if so, whether the antioxidant ebselen alleviates this vascular dysfunction. Experimental Approach: Male BALB/c mice were exposed to either room air or CS for 8 weeks followed by inoculation with IAV (Mem71, 1 x $104.5 \mathrm{pfu})$ ). Mice were treated with ebselen $(10 \mathrm{mg} / \mathrm{kg})$ or vehicle $(5 \% \mathrm{w} / \mathrm{v}$ CM-cellulose in water) daily. Mice were culled 3- and 10-days post-infection, and their lungs lavaged to assess inflammation. The thoracic aorta was excised to investigate endothelial and smooth muscle dilator responses, expression of key vasodilatory and oxidative stress modulators and infiltrating immune cells. Key Results: CS increased lung inflammation and caused significant vascular endothelial dysfunction, which was worsened by IAV infection. CS-driven increases in vascular oxidative stress and suppression of eNOS were not affected by IAV infection. CS and IAV infection significantly enhanced T-cell recruitment into the aortic wall. Ebselen abolished the exaggerated lung inflammation, vascular dysfunction and increased aortic T-cell infiltration in CS and IAV-infected mice. Conclusion and Implications: Ebselen abolished vascular dysfunction in IAV-induced exacerbations of cigarette smoke-induced lung inflammation indicating it may have potential for the treatment of cardiovascular comorbidities seen in acute exacerbations of COPD.
\end{abstract}

\section{Hosted file}

Brassington K AECOPD Ebselen final submitted 21102021.docx available at https://authorea. com/users/356125/articles/543169-ebselen-abolishes-vascular-dysfunction-in-influenza-a-

virus-induced-exacerbations-of-cigarette-smoke-induced-lung-inflammation-in-mice 
Figure 1
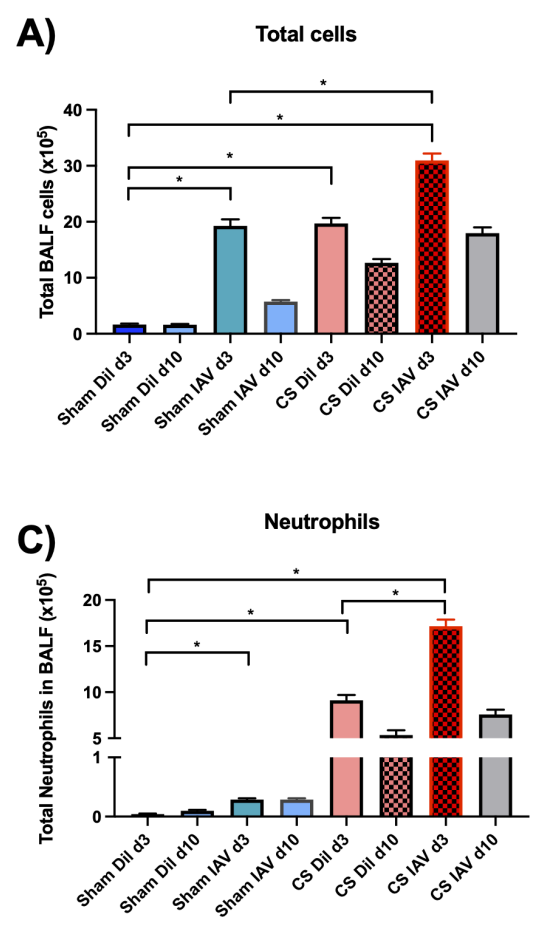
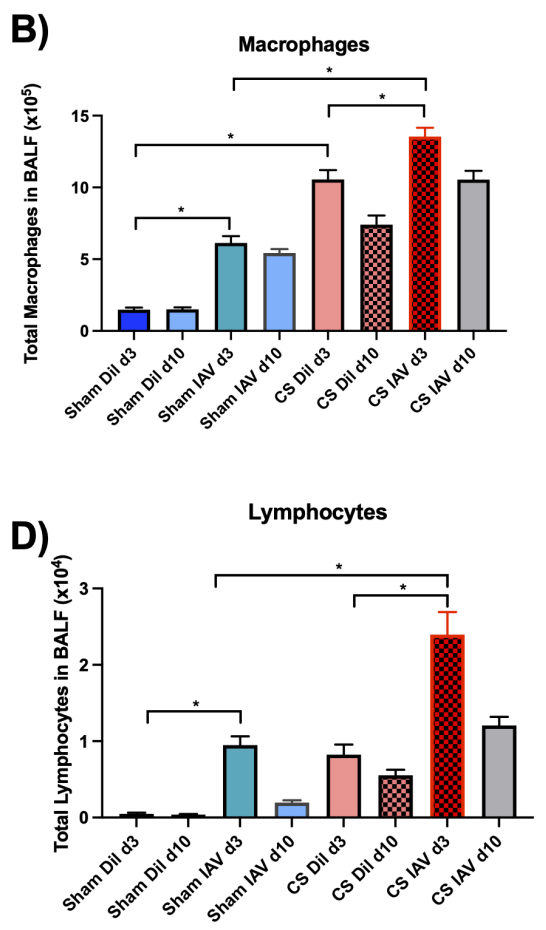
Figure 2

A)

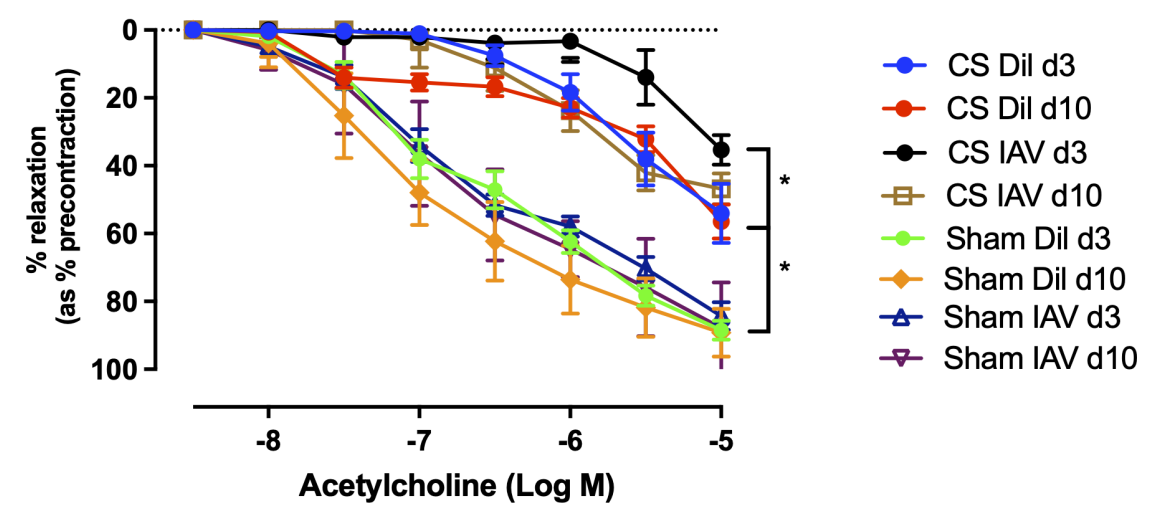

B)

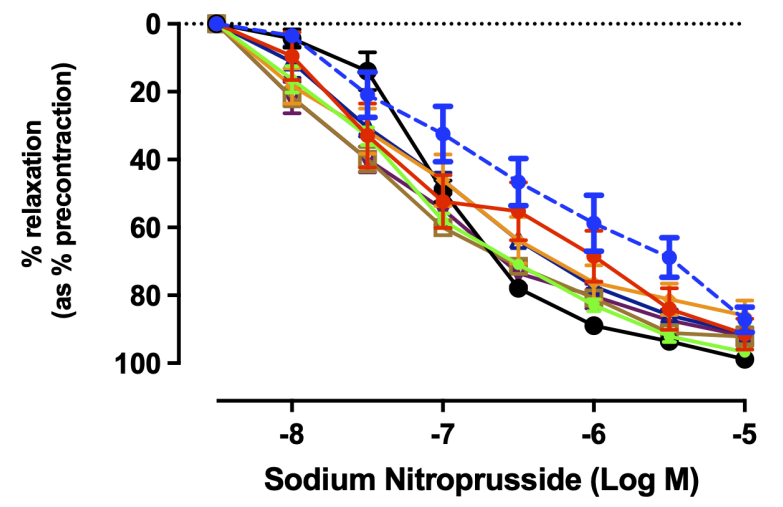


Figure 3

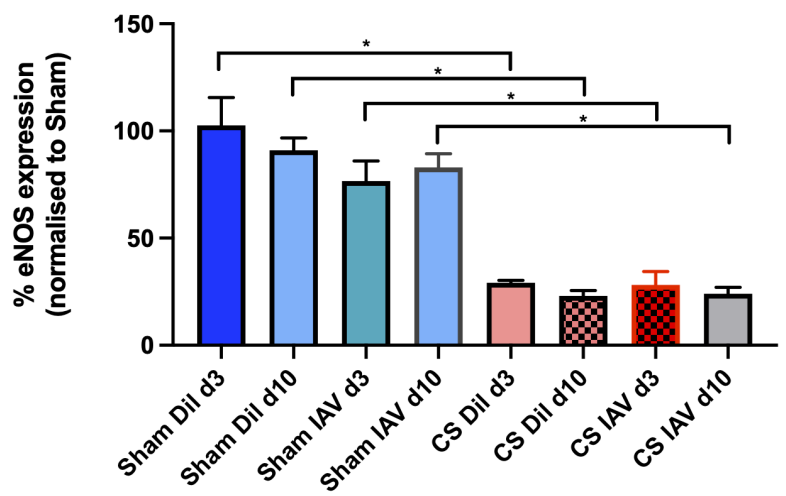

Dil d3

Dil d10

IAV d3

IAV d10

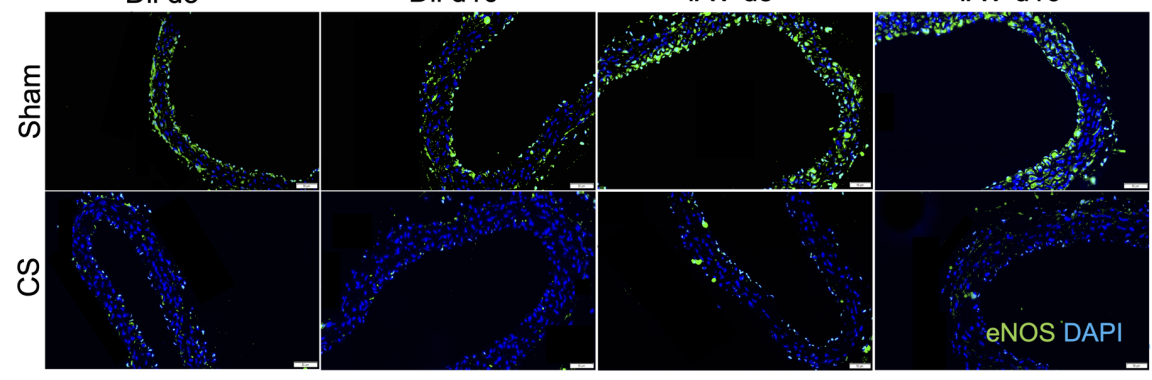


Figure 4

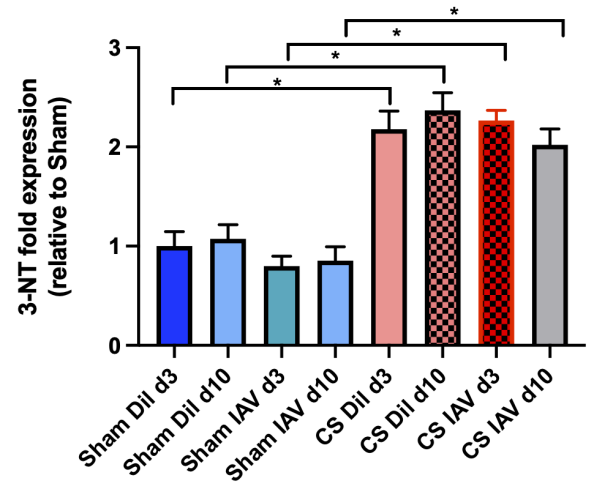

Dil d3

Dil d10

IAV d3

IAV d10

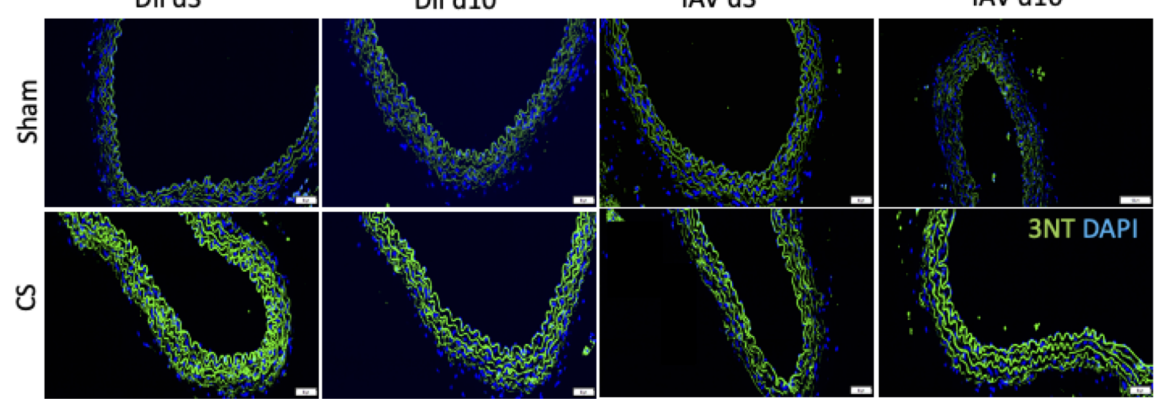


Figure 5

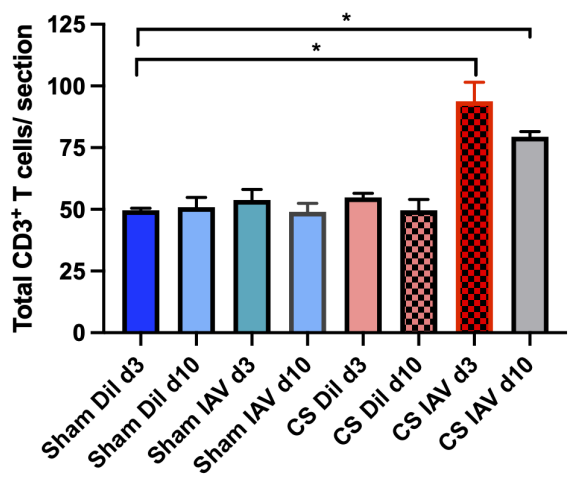

Dil d3

Dil d10

IAV d3

IAV d10

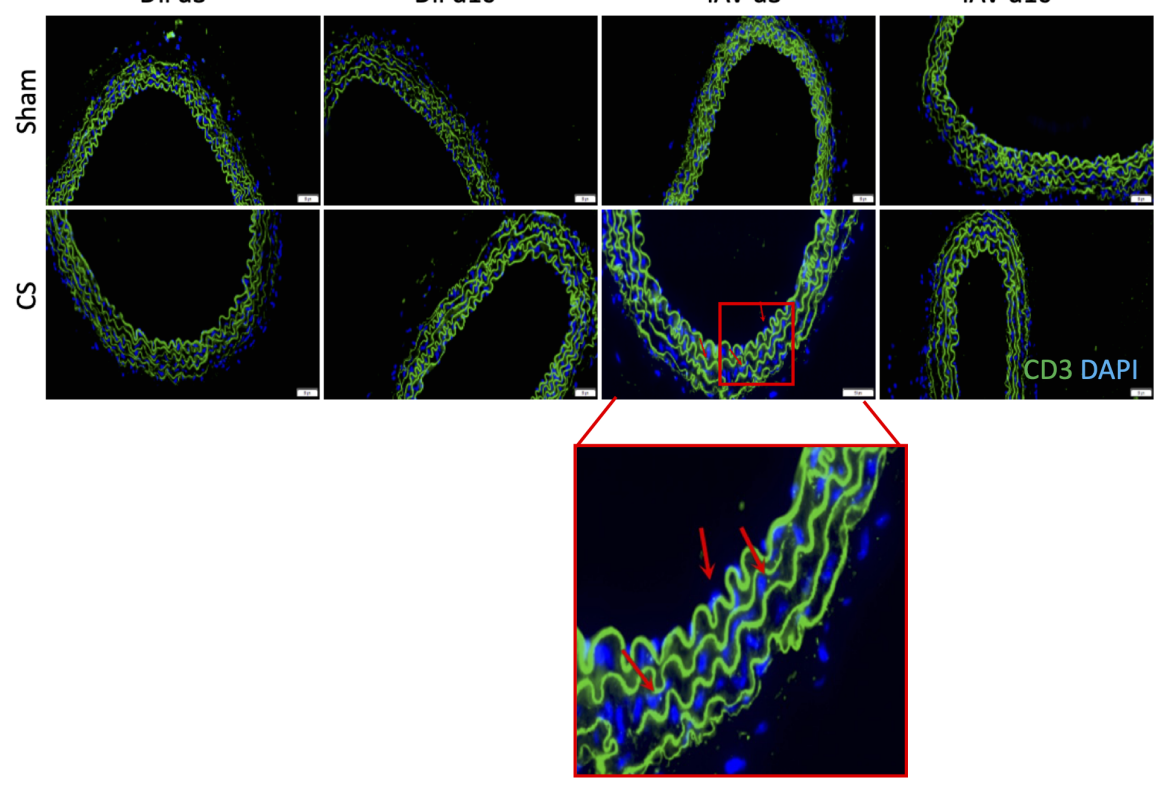


Figure 6

A)

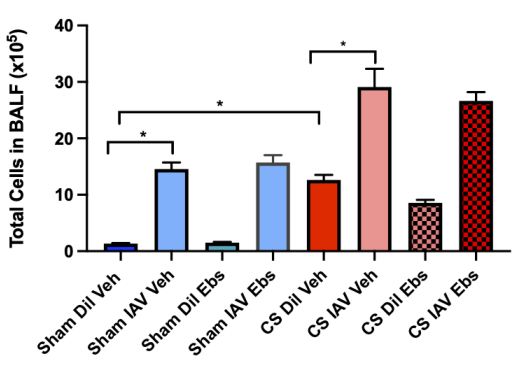

C)

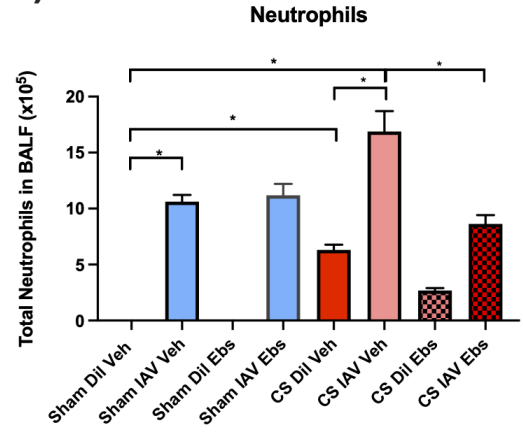

B)

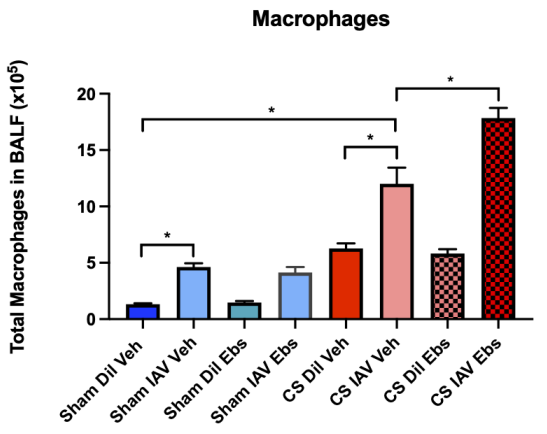

D)

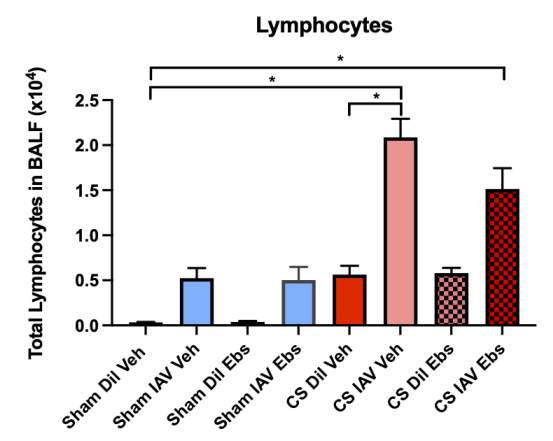


Figure 7

A)
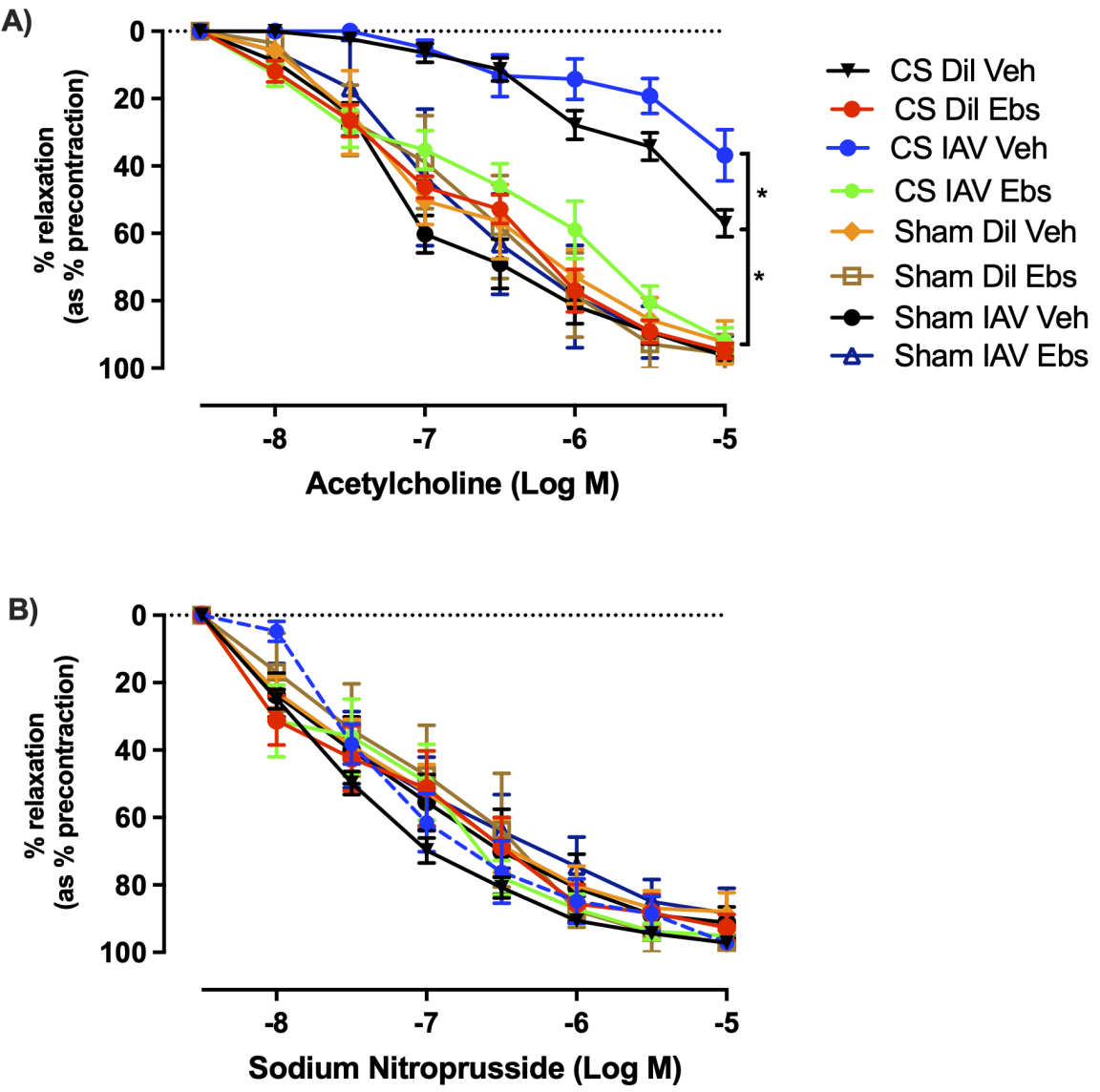
Figure 8
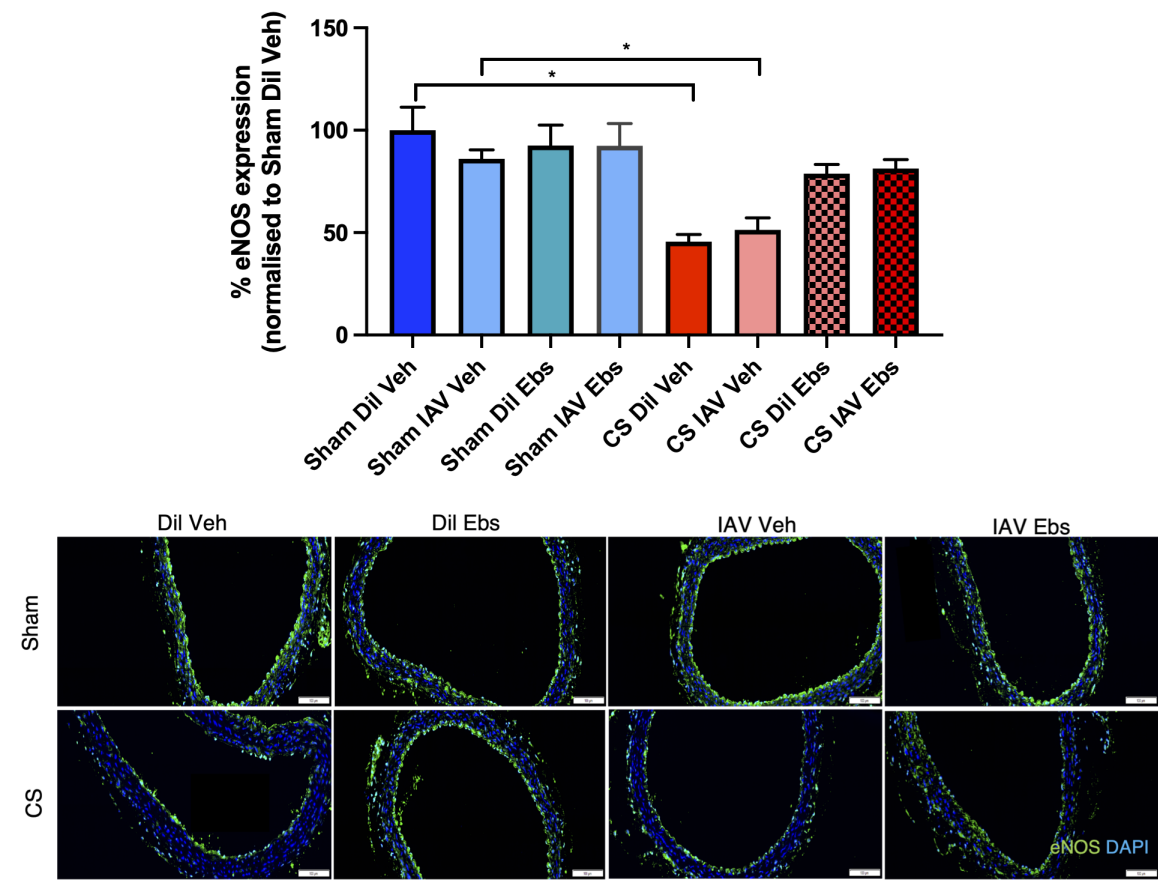

RMIT Classification: Trusted

Figure 9
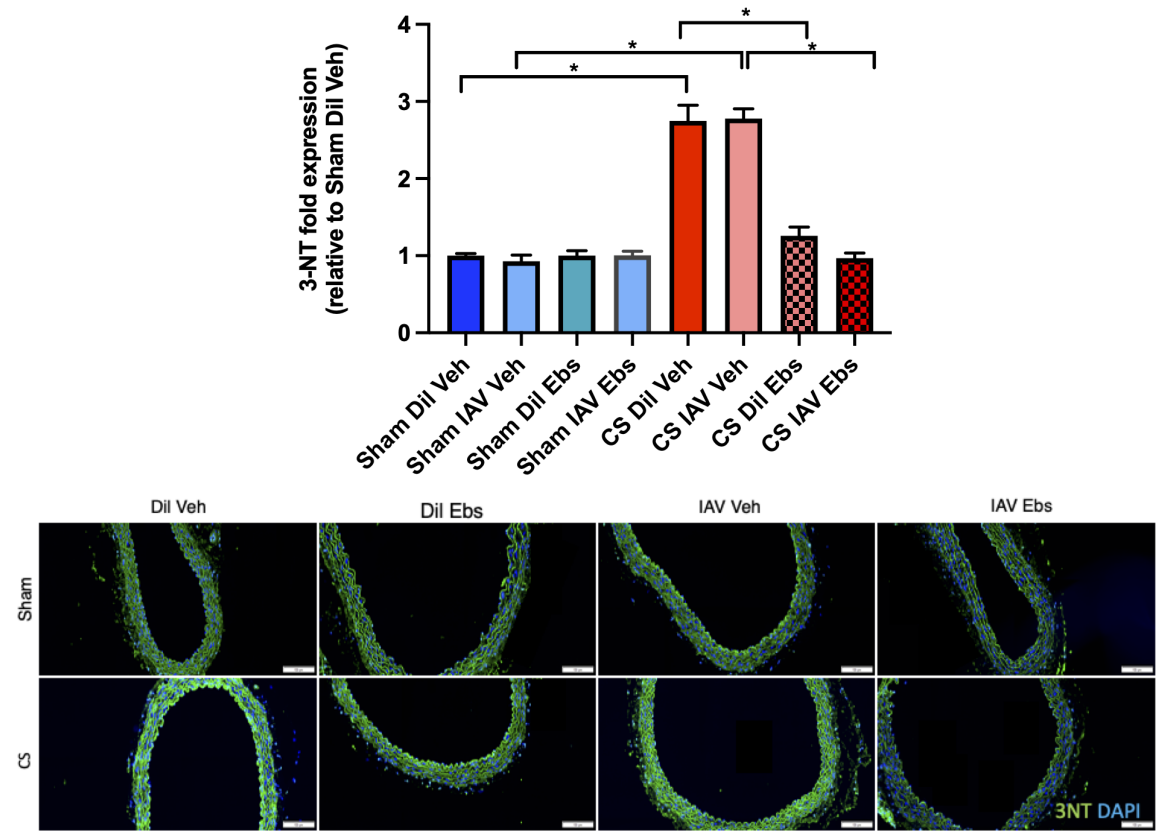
Figure 10
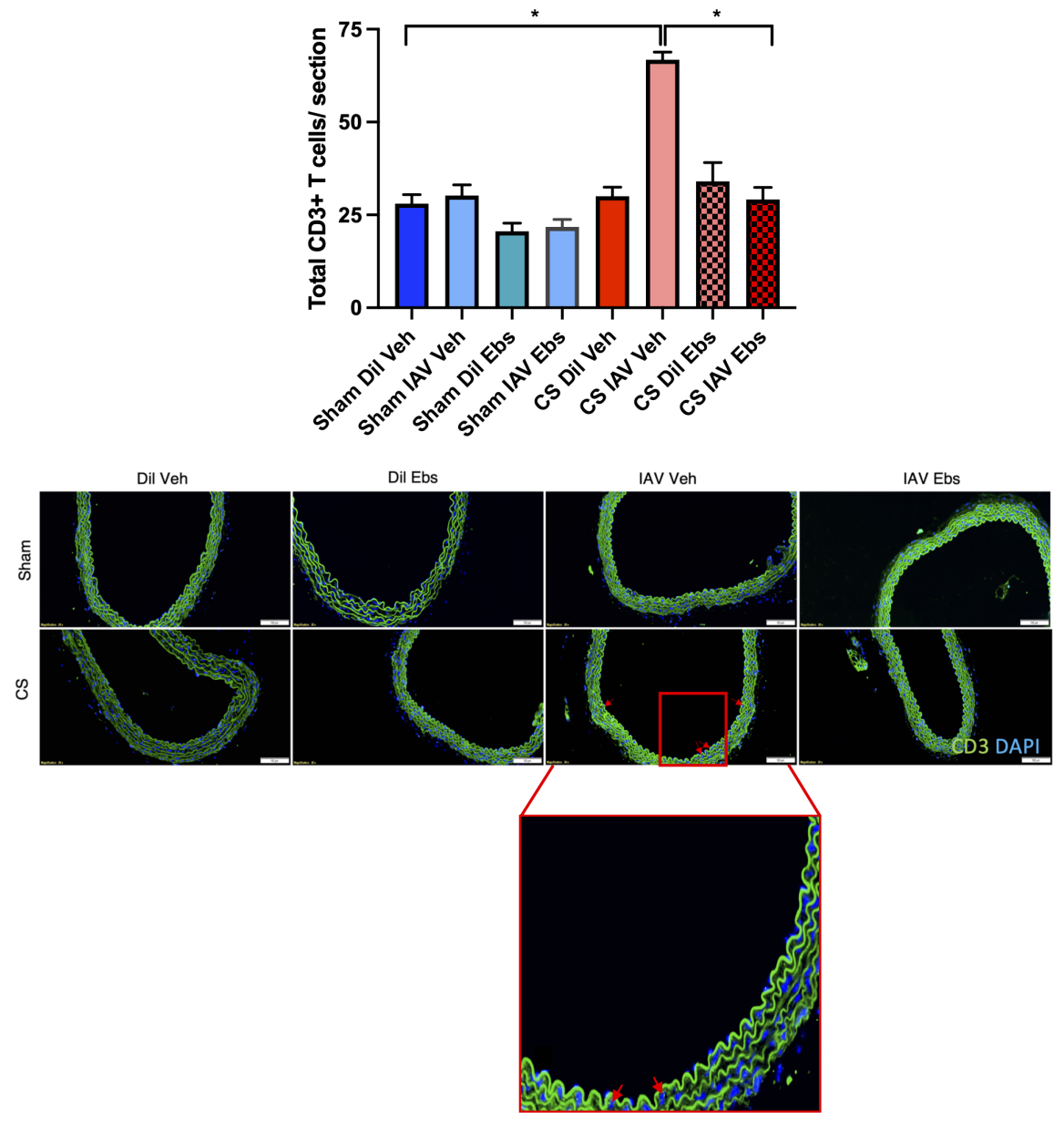